\title{
An in silico evaluation of Tn916 as a tool for generalized mutagenesis in Haemophilus influenzae Rd
}

\author{
Sarah L. Hosking, ${ }^{1}$ Mary E. Deadman, ${ }^{2}$ E. Richard Moxon, ${ }^{2}$ \\ John F. Peden, ${ }^{3}$ Nigel J. Saunders ${ }^{2}$ and Nicola J. High ${ }^{1}$ \\ Author for correspondence: Nicola J. High. Tel: +44 161275 5749. Fax: +44 1612755656. \\ e-mail: Nicky.High@man.ac.uk
}

1 University of Manchester, School of Biological Sciences, 1.800 , Stopford Building, Oxford Road, Manchester M13 9PT, UK

2 Molecular Infectious Diseases Group, University of Oxford, Institute of Molecular Medicine, John Radcliffe Hospital, Headington, Oxford OX3 9DS, UK

3 Molecular Biology Data Centre, IMSU, University of Oxford, Headington, Oxford, UK

\begin{abstract}
The transposon Tn916 was evaluated as a tool for generalized mutagenesis of the genome of Haemophilus influenzae. This was achieved in silico by searching the genome sequence of $H$. influenzae Rd for the published Tn916 target site consensus sequence $5^{\prime}$ TT/ATTIT(N) ${ }_{6}$ AAAAAATA. This search identified 16 putative target sites. In subsequent experiments, integration of Tn916 did not occur at any of these sites. Using the nucleotide sequences of these observed integration sites, a new consensus sequence, $5^{\prime}$ $\pi T I(N)_{x} A A A A A(4 \leq x \leq 7)$, was derived. This sequence reflects the curve-twist-curve DNA topology which is a feature common to all Tn916 integration sites. A search of the $H$. influenzae Rd genome using the new consensus sequence identified 167 potential target sites, representing approximately $1 \%$ of the total genome. Only 80 of these sites were located within ORFs. The presence of such a limited number of target sites places severe constraints on the use of Tn916 as a tool for generalized mutagenesis of the genome of $\boldsymbol{H}$. influenzae.
\end{abstract}

Keywords: Haemophilus influenzae Rd, Tn916 mutagenesis, genome sequence

\section{INTRODUCTION}

Haemophilus influenzae is a cause of both upper and lower respiratory tract infections (Turk, 1984). Strains expressing capsular serotype $\mathrm{b}$ also cause invasive disease such as meningitis. Recently, the complete genome sequence of $H$. influenzae $\mathrm{Rd}$, an avirulent, nonencapsulated derivative of a serotype $d$ strain, was determined. This information represents a powerful tool with which to study the biology of this organism (Fleischmann et al., 1995). Identification of ORFs in the $H$. influenzae Rd genome has been achieved by comparison of their nucleic acid and amino acid sequences with those of known genes and proteins. Of the 1743 predicted coding sequences identified in $H$. influenzae $\mathrm{Rd}, 736$ have not been assigned a function. No database matches have been found for 389 of these, whilst 347 have homology to hypothetical proteins which occur in other organisms. Thus, the function of many genes remains undetermined.

The role of key virulence determinants in the development of invasive disease, such as capsule and LPS, have been well characterized. However, invasive disease is a complex process and there are many steps which remain to be explored. To further characterize this process, methods are required to generate a representative set of mutants from which those with increased or decreased virulence can be selected. One strategy which is traditionally considered to fulfil this criterion is transposon mutagenesis. Naturally occurring transposons have not been identified in Haemophilus species. However, transposon Tn916 has been successfully introduced into the genomes of both $H$. influenzae and $H$. parainfluenzae by transformation using the suicide plasmid pAM120 (Kauc \& Goodgal, 1989) and by conjugation (Holland et al., 1992). Tn916 is an $18 \mathrm{~kb}$ conjugative transposon originally identified in the chromosome of Enterococcus faecalis (Franke \& Clewell, 1981). It is a member of a family of closely related conjugative transposons which includes Tn1545 from Streptococcus pneumoniae (Courvalin \& Carlier, 1987). These transposons are promiscuous and are able to function in a wide range of bacteria (Clewell et al., 1995). They have been used successfully to generate mutants deficient in both LPS and capsule biosynthesis in Neisseria meningitidis (Stephens et al., 1991; Kahler et al., 1996). Although Tn916 is capable of transposition 
in H. influenzae, it does not generate the comprehensive range of mutations typical of a transposon mutagenesis experiment. When this strategy was used to identify genes involved in LPS biosynthesis, mutations in genes directly involved were not recovered, although a mutant with an altered LPS phenotype was generated (High et al., 1996). This suggested that the number of sites into which Tn916 could integrate in the $H$. influenzae genome was limited, although the production of mutants with the required phenotype was possible.

Integration of Tn916 into host DNA has been reported to occur at target sequences with the consensus $5^{\prime}$ TT/ATTTT $(N)_{6}$ AAAAAA/TA, where $(N)_{6}$ represents any 6-base sequence (Lu \& Churchwood, 1995). This consensus was derived by examination of 24 target sites (compiled by Scott et al., 1994) used by Tn916 in Streptococcus pyogenes, Listeria monocytogenes, $B a-$ cillus subtilis, Ent. faecalis and N. meningitidis. This sequence has similarity with the nucleotide sequence of the right- (RH) and left-hand (LH) ends of Tn916. A characteristic of $\operatorname{Tn} 916$ target sequences is that they are predicted to contain static bends centred at the site of transposon insertion. This DNA topology is thought to facilitate binding of the integrase encoded by $\mathrm{Tn} 916$ and thereby integration of the transposon into the host genome (Lu \& Churchwood, 1995). No single base in the consensus integration sequence is conserved amongst all target sequences analysed to date and individual targets can differ from this common sequence at several positions whilst retaining the ability to interact with integrase. Since transposition of Tn916 is not a random event, its use as a tool for generating mutations in the genome of $H$. influenzae and other organisms will, consequently, be limited by the number and distribution of target sequences.

\section{METHODS}

Bacterial strains, culture conditions and plasmids. $H$. influenzae $\mathrm{Rd}$ is an avirulent, non-encapsulated derivative of a serotype d strain (Leidy et al., 1960). Escherichia coli strain CG120 contains the chimeric plasmid pAM120/pGL101 into which Tn916 has been cloned (Gawron-Burke \& Clewell, 1984).

$H$. influenzae strains were cultured in brain heart infusion (BHI) broth (Oxoid) supplemented with haemin $\left(10 \mu \mathrm{g} \mathrm{ml}^{-1}\right)$ and NAD $\left(2 \mu \mathrm{g} \mathrm{ml}^{-1}\right)$. Solid Levinthal's BHI medium was prepared by the addition of Bacto agar $(1 \%, w / v)$ and Levinthal base (Alexander, 1965). E. coli CG120 was grown in Luria broth supplemented with ampicillin $\left(25 \mu \mathrm{g} \mathrm{ml}^{-1}\right)$ and tetracycline $\left(4 \mu \mathrm{g} \mathrm{ml}^{-1}\right)$. H. influenzae Tn916 transformants were cultured in the presence of tetracycline $\left(4 \mu \mathrm{g} \mathrm{ml}^{-1}\right)$.

Transformation of $\boldsymbol{H}$. influenzae with Tn916. H. influenzae was transformed with pAM120 using the M-IV procedure (Herriot et al., 1970). In each transformation $1 \mu \mathrm{g}$ DNA was used to ensure that DNA was not limiting. Tn916 transformants were selected by growth on Levinthal's BHI medium containing tetracycline $\left(2 \mu \mathrm{g} \mathrm{ml}^{-1}\right)$. A transformation efficiency of $3.5 \times 10^{-3}$ was routinely achieved.

PCR amplification of Tn916-chromosomal DNA junction regions. Recognition sites for the restriction enzyme Sspl

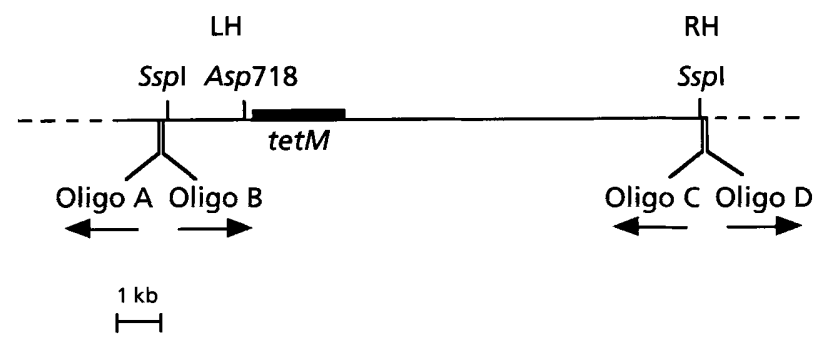

Fig. 1. Restriction map of Tn916 showing the relative positions of the Sspl and Asp718 restriction endonuclease sites used in this study. The position and direction of the primers used for PCR amplification of the LH and RH Tn916-chromosomal DNA junction regions are shown. The box indicates the location of tet $M$, the tetracycline resistance gene present in Tn916. The dashed lines either side of Tn916 represent $H$. influenzae chromosomal DNA.

occur frequently in the genome of $H$. influenzae, and 1613 and $203 \mathrm{bp}$ in from the $\mathrm{LH}$ and RH extremities of Tn916, respectively (Fig. 1). By digesting chromosomal DNA from each mutant with $S s p I$, two small restriction fragments harbouring the chromosomal DNA-Tn916 junctions were generated. The digested chromosomal DNA was then ligated under conditions that favoured intramolecular ligation. The ligated DNA was used as the template in an inverse PCR and the nucleotide sequence of the amplified product determined. The nucleotide sequences of the primers used to amplify the junction between chromosomal DNA and the LH extremity of Tn916 were 5' GAAGTATCTTCCTACAGT (Oligo A) and $5^{\prime}$ CCTCTGCCAAGCGTCGTAGC (Oligo B). 5' TTTGATACGACGATTTATCCT (Oligo C) and 5' TGATAAAGTGTGATAAGTCCA (Oligo D) were used to amplify the junction between chromosomal DNA and the RH extremity of Tn916 (Fig. 1). Primers A and C were biotinylated.

Direct sequencing of PCR products. Biotinylated PCR products were bound to streptavidin-coated Dynabeads (Dynal). The bound PCR product was denatured with $150 \mathrm{mM} \mathrm{NaOH}$, washed three times in sterile distilled water and then resuspended in $14 \mu \mathrm{l}$ distilled water. An aliquot $(7 \mu \mathrm{l})$ of this Dynabead-bound PCR product was then used for sequencing which was performed as outlined in the USB Sequenase handbook.

\section{RESULTS}

\section{The number and distribution of potential Tn916 target sites on the genome of $\boldsymbol{H}$. influenzae}

To assess whether Tn916 transposition is an effective method for generating mutations in $H$. influenzae, the number and distribution of potential Tn916 target sites on the genome of $H$. influenzae $\mathrm{Rd}$ was determined. The genome sequence was searched for sequences which matched the Tn916 target site consensus sequence, $5^{\prime}$ TT/ATTTT $(\mathrm{N})_{6}$ AAAAAA/TA, using the EGCG program FindPatterns which locates short nucleotide sequence patterns (Devereux et al., 1984; http:// www.sanger.ac.uk/Software/EGCG). Only 16 sequences which matched this consensus were identified. Since the target site consensus sequence does not represent every potential target sequence at which $\operatorname{Tn} 916$ 


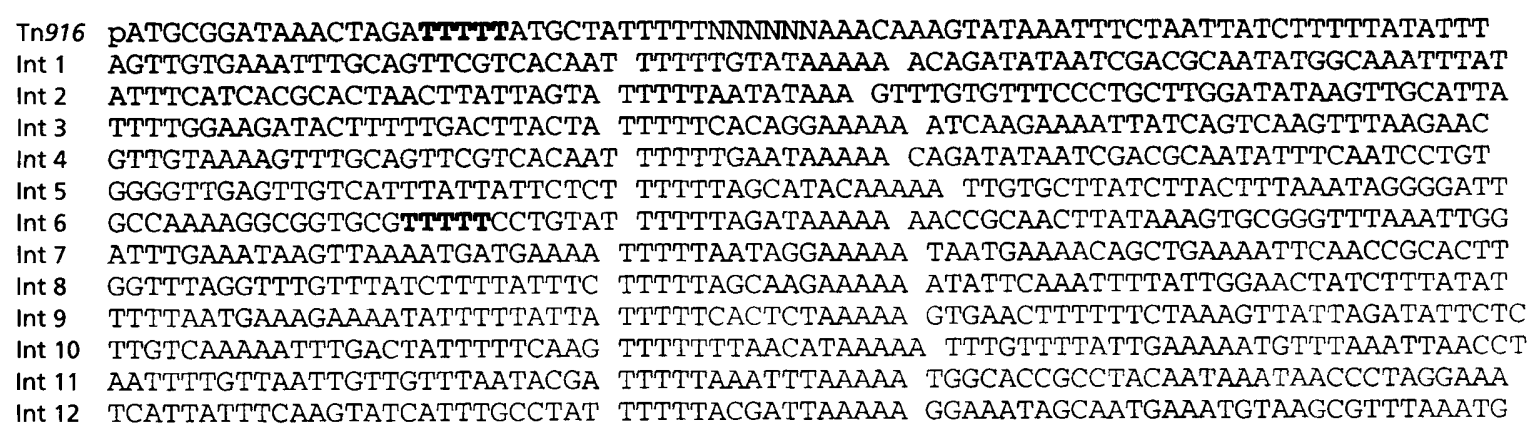

Fig. 2. Nucleotide sequences of the LH-RH junction region of the circular intermediate form of Tn916 and the integration sites of Tn916 identified in this study. The region corresponding to the consensus sequence $5^{\prime}$ TTITT(N) AAAAA $^{\prime}$ $(4 \leqslant x \leqslant 7)$ is separated from the main body of the sequence by a single space. NNNNNN represents the variable coupling sequence present in Tn916. The additional tract of $5 \mathrm{dT}$ residues present in the LH-RH junction region of the circular intermediate form of Tn916 and in target site Int 6 is highlighted in bold.

can insert, this figure is only a conservative estimate of the number of such sites on the genome of $H$. influenzae. Nevertheless, it indicated that potential Tn916 integration sites were not widely represented.

\section{Identification of Tn916 integration sites in $H$. influenzae}

To test whether the above prediction was accurate, it was necessary to determine how effective the consensus sequence was in identifying $\operatorname{Tn} 916$ integration sites in $H$. influenzae. To achieve this Tn916 was introduced onto the genome of $H$. influenzae in two independent transformation experiments. Eighteen different transformants were selected arbitrarily, without regard for any alteration in phenotype and the relative location of Tn916 in each was determined by Southern hybridization. Genomic DNA from each transformant was digested with Asp718 which cuts once in Tn916 and $X b a \mathrm{I}$ that does not cut within the transposon. This generated two DNA fragments containing the LH and RH ends of Tn916 and the associated flanking DNA. Digested genomic DNA from each transformant was then probed with $\operatorname{Tn} 916$. Twelve distinct hybridization patterns were apparent in the 18 transformants analysed (data not shown), suggesting that $\mathrm{Tn} 916$ had integrated into only 12 different genomic locations. In all cases each mutant only contained one copy of the transposon. One hybridization pattern occurred a total of five times in both transformation experiments, suggesting that Tn916 had inserted into an integration 'hot-spot'.

\section{Determination of the nucleotide sequence of Tn916 integration sites}

The site at which $\mathrm{Tn} 916$ had integrated into the genome of each mutant was determined by sequencing the Tn916-chromosomal DNA junctions (Fig. 2). By comparison of each sequence to the genome sequence of $H$. influenzae $\mathrm{Rd}$, the location of each target site and the identity of any ORF that had been disrupted was established (Table 1). As suggested by Southern hybri- dization, only 12 different $\operatorname{Tn} 916$ insertion mutants had been generated. Of these, 10 were located within intergenic regions (Table 1). A total of five Tn916 insertions occurred at the target site Int 6 in two independent transformation experiments. Three of these insertions were derived from the first transformation and the remaining two from the second transformation. Two insertions at target sites Int 5 and Int 8 were observed. In the case of Int 5 , both insertions were obtained from the same transformation. Given that a transformation efficiency of $3.5 \times 10^{-3}(\mathrm{mg} \mathrm{DNA})^{-1}$ was routinely obtained and the cells were only incubated for 20 min prior to plating out on selective media, it is unlikely that the two Int 5 transformants are siblings.

\section{Searching the $\boldsymbol{H}$. influenzae Rd genome sequence with a modified Tn916 target site consensus sequence}

None of the target sites identified in this study matched the published consensus sequence. A consensus sequence reflecting the characteristics of the integration sites identified in this study was therefore derived to obtain a more accurate estimate of the number and location of potential Tn916 target sites in the Rd genome.

Each of the Tn916 integration sites was analysed using the programs DNA + and RasMol which calculate the phase and degree of curvature of a nucleotide sequence (DeSantis et al., 1996; Sayle \& Milner-White, 1995). All of the integration sites were predicted to form curved DNA structures containing a phase change, resulting in a curve-twist-curve structure (Fig. 3). The curve in the target sites is generated by the short tracts of $\mathrm{dA}$ and $\mathrm{dT}$ on either side of the central $(\mathrm{N})_{6}$ sequence. When these occur on different faces of the helix, the overall direction of curvature, or phase, is different on either side of the coupling sequence, introducing a twist into the helix. Int 6 , at which five Tn916 insertions had occurred, was predicted to have the greatest DNA curvature due to the presence of an additional homopolymeric tract of $5 \mathrm{dT}$ residues with preserved helical facing, located $6 \mathrm{bp} 5^{\prime}$ to 
Table 1. The location of each transposon integration site on the $H$. influenzae Rd genome

\begin{tabular}{|c|c|c|c|c|c|}
\hline $\begin{array}{l}\text { Target } \\
\text { site }\end{array}$ & $\begin{array}{l}\text { Location of } \\
\text { insertion on the } \\
\text { Rd genome }\end{array}$ & $\begin{array}{c}\text { ORF } \\
\text { disrupted }\end{array}$ & & Identity of nearest ORFs & $\begin{array}{c}\text { Location of } \\
\text { ORFs on genome }\end{array}$ \\
\hline \multirow[t]{2}{*}{ Int 1} & \multirow[t]{2}{*}{$1317346-1317410$} & \multirow[t]{2}{*}{ No } & HI1244 & No database match & $1317246-1316629$ \\
\hline & & & HI1245 & Malic acid enzyme & $1317444-1319711$ \\
\hline Int 2 & $387337-387411$ & Yes & HI0364 & Penicillin-binding protein 5 precursor & $387935-387060$ \\
\hline \multirow[t]{2}{*}{ Int 3} & \multirow[t]{2}{*}{$1727530-1727605$} & \multirow[t]{2}{*}{ No } & HI1660 & Ribonucleoside diphosphate reductase & $1726333-1727460$ \\
\hline & & & HI1661 & Dihydrolipoamide succinyltransferase & $1728808-1727582$ \\
\hline \multirow[t]{2}{*}{ Int 4} & \multirow[t]{2}{*}{$1317347-1317422$} & \multirow[t]{2}{*}{ No } & HI0985 & DNA processing chain & $1042260-1041085$ \\
\hline & & & HI0986 & Isopropylmalate synthetase & $1042715-1044307$ \\
\hline \multirow[t]{2}{*}{ Int 5} & \multirow[t]{2}{*}{$1353302-1353375$} & \multirow[t]{2}{*}{ No } & HI1276 & Methionine tRNA synthetase & $1353315-1351270$ \\
\hline & & & HI1277 & Putative ATPase (Mrp) & $1353418-1354575$ \\
\hline \multirow[t]{2}{*}{ Int 6} & \multirow[t]{2}{*}{$1042650-1042710$} & \multirow[t]{2}{*}{ No } & HI0985 & DNA processing chain & $1042206-1041085$ \\
\hline & & & HI0986 & Isopropylmalate synthetase & $1042715-1044307$ \\
\hline \multirow[t]{2}{*}{ Int 7} & \multirow[t]{2}{*}{$1218830-1218900$} & \multirow[t]{2}{*}{ No } & HI1151 & Hypothetical protein & $218780-1218824$ \\
\hline & & & HI1152 & PmbA & $1218869-1220221$ \\
\hline \multirow[t]{2}{*}{ Int 8} & \multirow[t]{2}{*}{$1808470-1808510$} & \multirow[t]{2}{*}{ No } & HI733 & RNase II & $1810602-1807374$ \\
\hline & & & HI732 & AidA-1 adhesin & $1808267-1807374$ \\
\hline \multirow[t]{2}{*}{ Int 9} & \multirow[t]{2}{*}{$1616560-1616630$} & \multirow[t]{2}{*}{ No } & HI546 & Hypothetical protein & $1616761-1617180$ \\
\hline & & & HI547 & DAHP synthetase & $1618360-1617275$ \\
\hline \multirow[t]{2}{*}{ Int 10} & \multirow[t]{2}{*}{$581150-581250$} & \multirow[t]{2}{*}{ No } & HI10561 & Hypothetical protein & $581046-579148$ \\
\hline & & & HI10562 & Hypothetical protein & $581360-581752$ \\
\hline Int 11 & 209569-209644 & Yes & HI0195 & Hypothetical protein & $208082-211414$ \\
\hline \multirow[t]{2}{*}{ Int 12} & \multirow[t]{2}{*}{$892797-892893$} & \multirow[t]{2}{*}{ No } & HI10844 & Guanidine dinucleotide biosynthesis & $892784-892209$ \\
\hline & & & HI10845 & Hypothetical protein & $892871-893134$ \\
\hline
\end{tabular}

(a)

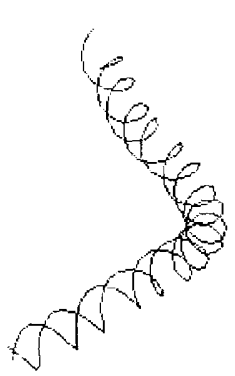

(b)

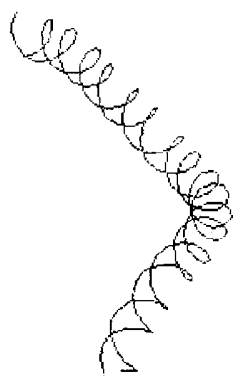

(c)

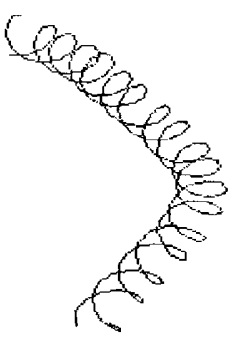

Fig. 3. DNA secondary structure formed by (a) integration site Int 6 (angle of curvature $131^{\circ}$ ), (b) the Tn916 LH-RH junction region of the circular intermediate form of $\operatorname{Tn} 916\left(121^{\circ}\right)$ and (c) integration site Int $2\left(138^{\circ}\right)$. The nucleotide sequence used to generate this data is illustrated in Fig. 2. The graphic output and the degree of DNA curvature of each target site was obtained using the program RasMol (Sayle \& Milner-White, 1995).

the TTTTTT $(\mathrm{N})_{6}$ AAAAA sequence. An equivalent homopolymeric tract is also present at the same position in the Tn916 LH-RH junction region (Fig. 2). The presence of this sequence would have the effect of increasing the degree of DNA curvature within this region relative to other target sites. This observation may explain why Int 6 acts as a 'hot-spot' for Tn916 insertion.

In 8 of the 12 Tn916 integration sites the curvetwist-curve structure coincided with the presence of the motif $5^{\prime}$ TTTTTT $(\mathrm{N})_{6}$ AAAAA at the centre of the integration site, where $(N)_{6}$ represents the coupling sequence and is composed of any base. In the remaining sites it is formed by equivalent tracts of $\mathrm{dA}$ and $\mathrm{dT}$ separated by either 4,5 or $7 \mathrm{nt}$. When the contribution of the polyA and polyT tracts to the observed curvature is considered, the sequence $5^{\prime}$ TTTTT $(\mathrm{N})_{\mathrm{x}}$ AAAAA $(4 \leqslant x \leqslant 7)$ more accurately describes the determinants of local topology associated with Tn916 integration sites. Only one integration site, Int 2, did not fit this pattern, although it was predicted to form a similar secondary structure to the other integration sites identified (Fig. 3). Comparison to the consensus sequence derived by Lu \& Churchwood (1995) revealed that our modified consensus matched $50 \%$ of the 16 target sites that it had identified. When the $H$. influenzae Rd genome sequence was searched with the new consensus, 167 matches were found of which 80 were located in ORFs.

\section{DISCUSSION}

Although it is well established that the transposon Tn916 can integrate into the genome of $H$. influenzae, it has not proven to be an effective tool for generating mutations with defined phenotypes in this organism. This has led to the suggestion that the number of integration sites for Tn916 in the genome of $H$. influenzae might be limited. Experiments carried out by 
Kauc \& Goodgal (1989) determined the location of Tn916 integration sites on the genome of $H$. influenzae Rd by agarose gel electrophoresis of ApaI- and SmaIdigested genomic DNA. Although this strategy confirmed that Tn916 could integrate into the $H$. influenzae genome, the precise location of each integration site could not be defined. The completion of the $H$. influenzae $\mathrm{Rd}$ genome sequence provided the first opportunity to determine the number and relative distribution of Tn916 integration sites in this organism. Only 16 potential Tn916 target sites which fitted the Tn916 integration site consensus sequence (Lu \& Churchwood, 1995) were identified. These sites were not used by Tn916 in subsequent transformation experiments, indicating that it could integrate into the $\mathrm{Rd}$ genome at sites which did not fit the consensus sequence. To obtain a more accurate estimate of the number of potential target sites, a new, more comprehensive consensus sequence was derived through comparison of the actual target sites used by the transposon in $H$. influenzae.

A feature of the Tn916 integration sites identified in this study is that they are predicted to form a characteristic curve-twist-curve secondary structure. A similar structure is also formed by the LH-RH junction of Tn916 which binds directly to integrase. DNA topology may therefore play an important role in mediating integration of Tn916 into host DNA. A similar curve-twist-curve structure is also an important component of the target sites used by the Bacillus subtilis insertion element IS231 A, although in this case the structure is formed by tracts of poly-dC and poly-dG (Hallet et al., 1994). The extent of DNA curvature also appears to influence the frequency with which sites are used as targets for Tn916 integration. Tn916 appears to insert preferentially into target sites such as Int 6 which have a relatively high degree of DNA curvature. The DNA curvature at the Int 6 integration site is similar to that formed by the Tn916 LH-RH junction region which is involved in binding integrase (Fig. 3). The secondary structure of Int 6 may therefore result in an optimal interaction with integrase which would explain why Tn916 integration occurs here more frequently than at other target sites. The affinity with which a target site binds integrase and hence the frequency with which it experiences Tn916 insertion may therefore be dictated in part by its secondary structure (Lu \& Churchwood, 1995).

Since the Tn916 integration sites identified in $H$. influenzae have a conserved DNA topology, it was concluded that a consensus sequence reflecting this feature might identify a more comprehensive range of integration sites. Based on this criterion the consensus sequence $5^{\prime}$ TTTTT $(\mathrm{N})_{\mathrm{x}}$ AAAAA $(4 \leqslant x \leqslant 7)$, was derived that fitted all but one of the integration sites, the exception being Int 2 which contains only $3 \mathrm{dA}$ residues. When this sequence was used to search the H. influenzae Rd genome, 167 potential Tn916 integration sites were identified. Since there are target sites, such as Int 2, which do not fit the consensus but have a secondary structure permissive for Tn916 integration, we assume that the number of actual sites on the genome would be larger.

Of the Tn916 integration sites identified, 80 occurred in intergenic regions. This number was larger than expected since non-coding regions account for only $13 \%$ of total genomic DNA. This observation indicated that the distribution of $\mathrm{Tn} 916$ target sites on the Rd genome was biased. If the target sites had been randomly distributed then 145 of them would be expected to occur in ORFs. This disproportionate distribution of target sites was also reflected in the low frequency with which Tn916 insertions within ORFs were isolated experimentally. In general, intergenic regions of the $H$. influenzae genome have a higher $\mathrm{A}+\mathrm{T}$ content than coding regions, which may reflect the fact that such regions harbour operator sequences. As a consequence, intergenic regions are therefore more likely to contain sequences which meet the criteria of the A+T-rich Tn916 target sites. The disruption of such regions may not, however, always result in a phenotype. The limited number of Tn916 integration sites in the genome of $H$. influenzae, in conjunction with their tendency to occur in non-coding regions, emphasizes the unsuitability of Tn916 as a tool for generalized mutagenesis of $H$. influenzae. It should be stressed, however, that the isolation of specific mutants using Tn916 is not impossible and that this system has been used to isolate mutations in iron regulatory genes (Holland et al., 1992). The success of this strategy will, however, inevitably be dictated by whether genes of interest contain a Tn916 target site.

In summary we have demonstrated that the number sites at which Tn916 can integrate into the genome of $H$. influenzae is limited. Integration sites occur predominately in intergenic regions and consequently may not always lead to an altered phenotype. It is therefore concluded that Tn916 is not suitable a tool for generalized mutagenesis of $H$. influenzae. In addition to the specific analysis of the $\operatorname{Tn} 916$ integration sites in $H$. influenzae, this study also demonstrates how bacterial genome sequences not only facilitate the rapid identification of genes of interest from small amounts of sequence information, but allow the efficiency of potential mutagenesis tools for the investigation of an individual organism to be assessed.

\section{ACKNOWLEDGEMENTS}

This work has been funded by a Wellcome project grant awarded to N. J.H. and an MRC programme grant awarded to E.R.M. N.J.S. is supported by a Wellcome Research Training Fellowship in Medical Microbiology.

\section{REFERENCES}

Alexander, H. E. (1965). The Haemophilus group. In Bacterial and Mycotic Infections of Man, pp. 724-741. Edited by R. J. Dubos \& J. G. Hirsch. London: Pitman Medical Publishing.

Clewell, D. B., Flannagan, S. E. \& Jaworski, D. D. (1995). Unconstrained bacterial promiscuity: the $\operatorname{Tn} 916-\operatorname{Tn} 1545$ family of conjugative transposons. Trends Microbiol 3, 229-236. 
Courvalin, P. \& Carlier, C. (1987). Tn1545. A conjugative shuttle transposon. Mol Gen Genet 206, 259-264.

DeSantis, P., Fua, M., Savino, M., Anselmi, C. \& Bacchinofuso, G. (1996). Sequence dependent circularisation of DNA: a physical model to predict the DNA sequence dependent propensity to circularisation and its changes in the presence of protein induced bending. J Phys Chem 100, 9968-9976.

Devereux, J., Haeberli, P. \& Smithies, O. (1984). A comprehensive set of sequence analysis programs for the VAX. Nucleic Acids Res 12, 387-395.

Fleischmann, R. D., Adams, M. D., White, O. \& 37 other authors (1995). Whole-genome random sequencing and assembly of Haemophilus influenzae Rd. Science 269, 496-512.

Franke, A. E. \& Clewell, D. B. (1981). Evidence for a chromosomeborne resistance transposon ( $\mathrm{Tn}$ 916) in Streptococcus faecalis that is capable of 'conjugal' transfer in the absence of a conjugative plasmid. $J$ Bacteriol 145, 494-502.

Gawron-Burke, C. \& Clewell, D. B. (1984). Regeneration of insertionally activated streptococcal DNA fragments after excision of Tn916 in Escherichia coli: strategy for targeting and cloning genes from Gram positive bacteria. J Bacteriol 159, 214-221.

Hallet, B., Rezsohazy, R., Mahillon, J. \& Deicour, J. (1994). IS231 A insertion specificity: consensus sequence and DNA bending at the target site. Mol Microbiol 14, 131-139.

Herriot, R. M., Meyer, E. M. \& Vogt, M. J. (1970). Defined nongrowth media for stage II development of competence in Haemophilus influenzae. J Bacteriol 101, 517-524.

High, N. J., Deadman, M. E., Hood, D. W. \& Moxon, E. R. (1996). The identification of novel lipopolysaccharide genes in Haemophilus influenzae using transposon Tn916 mutagenesis. FEMS Microbiol Lett 145, 325-333.

Holland, J., Towner, K. J. \& Williams, P. (1992). Tn916 insertion mutagenesis in Escherichia coli and Haemophilus influenzae type b following conjugative transfer. J Gen Microbiol 138, 509-515.

Kahler, C. M., Carlson, R. W., Rahman, M. M., Martin, L. E. \& Stephens, D. S. (1996). Inner core biosynthesis of lipooligosaccharide (LOS) in Neisseria meningitidis serogroup B: identification and role in LOS assembly of the $\alpha-1,2 \mathrm{~N}$-acetylglucosamine transferase (RfaK). J Bacteriol 178, 1265-1273.

Kauc, L. \& Goodgal, S. H. (1989). Introduction of transposon Tn916 DNA into Haemophilus influenzae and Haemophilus parainfluenzae. J Bacteriol 171, 6625-6628.

Leidy, G., Hahn, E., Zamenhof, S. \& Alexander, H. E. (1960). Biochemical aspects of virulence of Haemophilus influenzae. Ann N Y Acad Sci 88, 1195-1202.

Lu, F. \& Churchwood, G. (1995). Tn916 target DNA sequences bind the C-terminal domain of integrase protein with different affinities that correlate with transposon insertion frequency. $J$ Bacteriol 177, 1938-1946.

Sayle, R. A. \& Milner-White, E. J. (1995). RasMol: biomolecular graphics for all. Trends Biochem Sci 20, 374.

Scott, J. R., Bringel, F., Marra, D., van Alstine, G. \& Rudy, C. K. (1994). Conjugative transposition of Tn916: preferred target sites and evidence for conjugative transfer of a single strand and for a double-stranded circular intermediate. Mol Microbiol 11, 10991108 .

Stephens, D. S., Swartley, J. S., Kathariou, S. \& Morse, S. A. (1991). Insertion of Tn916 in Neisseria meningitidis resulting in loss of Group B capsular polysaccharide. Infect Immun 59, 4097-4102.

Turk, D. C. (1984). The pathogenicity of Haemophilus influenzae. J Med Microbiol 18, 1-16.

Received 4 February 1998; revised 25 April 1998; accepted 14 May 1998. 